\title{
Enhancement of neutrino detection with Auger through oscillation
}

\section{Pierre Billoir*, Xavier Bertou, Olivier Deligny, Antoine Letessier-Selvon}

\author{
LPNHE, CNRS/Univ. Paris VI/Paris VII, Paris, France
}

E-mail: ibilioir@in2p3.fri

\begin{abstract}
Ultra-high energy neutrinos are expected, either as byproducts of interactions of accelerated charged particles with intermediate mediums (in bottom-up models), or in direct decay of particles from a supermassive object (in top-down models); in the latter case they are (together with the photons) dominant in the incident flux. Neutrinos come essentially from pion decay, and the tauic flavour is rare. A muonic/tauic oscillation with full mixing would result in an equipartition. Tauic neutrinos interacting in the rock produce penetrating tau leptons, which may decay just after emerging from the earth, and generate an atmospheric shower, detectable by the Auger Observatory if it is nearly horizontal. The sensitivity is maximal around $1 \mathrm{EeV}$, and much larger than with direct atmospheric neutrino interactions. Auger could put strong constraints on production models, and even be sensitive to the "almost certain" GZK neutrino flux.
\end{abstract}

\section{Introduction}

While the cosmic ray spectrum is now shown to extend beyond $10^{20} \mathrm{eV}$, mechanisms producing or accelerating particles with such energies are still uncertain. Only very powerful astrophysical objects can, in principle, produce ultra high energies (UHE) through conventional acceleration; moreover the accelerated particles have to escape the site without large energy losses, which is a severe restriction. Speculative hypotheses involving new physics such as collapse of Topological Defects (TD) or decay of Super Massive Relic Particles (SMRP) at the Grand Unification scale are well suited to produce UHE particles; these models predict a large production of UHE photons and neutrinos from meson decays.

Transport from the source to Earth is also an issue. At UHE the interactions with cosmic background radiation led Greisen, Zatsepin and Kuzmin [i] cutoff $(\mathrm{GZK})$ around $5 \times 10^{19} \mathrm{eV}$ for protons or nuclei, if the sources are at a distance more than a few $10 \mathrm{Mpc}$; the available data, although very scarce, do not support such a cutoff.

${ }^{*}$ Speaker. 
An important byproduct of these interactions are the so-called GZK neutrinos. Contrary to photons, neutrinos are essentially unaffected on their journey to Earth: they may allow us to disentangle the source characteristics from the propagation distortions.

We briefly describe the Auger observatory and show how $\nu_{\tau}$ may be detected as almost horizontal showers. Assuming full $\nu_{\mu} \leftrightarrow \nu_{\tau}$ mixing we evaluate our sensitivity to potential neutrinos sources. Previous studies on the observation of atmospheric interactions with

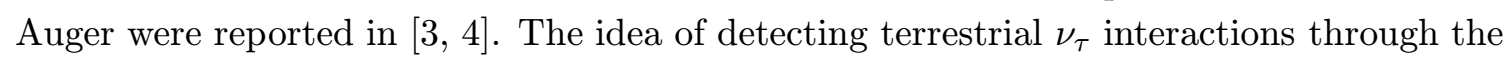

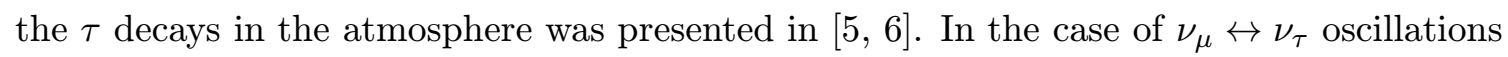
with full mixing [i] large distance between the source and the Earth. Unlike electrons (absorbed in earth) or muons (giving only a narrow electromagnetic halo), almost horizontal taus produced in the ground can decay after emerging and produce a detectable shower.

\section{Neutrino detection with a Ground Detector}

Large area ground based detectors observe the incident cosmic rays through the Extensive Air Showers (EAS), a huge cascade of particles generated throughout the atmosphere. The Auger Observatories ${ }^{1}[\overline{2}[\overline{2}]$ combine fluorescence telescopes (observation of the longitudinal profile) with ground array of Čerenkov water tanks (measurement of lateral densities).
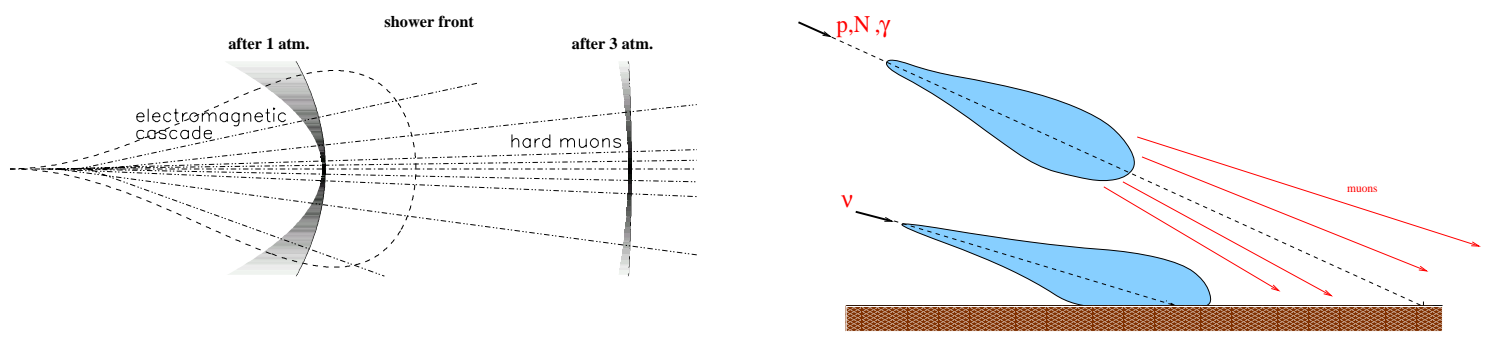

Figure 1: Development of a shower and neutrino discrimination.

The UHE neutrinos ${ }^{2}$ may be distinguished from ordinary hadrons by the shape of the shower they produce (see Figure $\left.\left.{ }_{1}^{1}\right]_{1}^{1}\right)$ : at large zenith angles (above 70 deg.) the electromagnetic part of ordinary showers is totally extinguished and only high energy muons survive; in addition, the shower front is very flat and the particles time spread is very narrow (less than $50 \mathrm{~ns}$ ). On the contrary, deeply induced showers have a curved front, a large electromagnetic component, and with particles well spread over time.

On the other hand, horizontal showers at low altitude, due to their longitudinal extension, may be seen in Auger at an energy much lower than vertical ones. For example, a primary particle of $0.1 \mathrm{EeV}$ gives an effective radius larger than $300 \mathrm{~m}$ over $10 \mathrm{~km}$. Fig. $\underline{2}$ shows examples of shower ground spots.

\footnotetext{
${ }^{1}$ Named after the French physicist Pierre Auger; the first one is being constructed in the southern hemisphere, the second one is planned in northern hemisphere

${ }^{2}$ We do not consider here questionable models invoked to explain the events above GZK cutoff, where the neutrinos undergo strong interactions at UHE.
} 

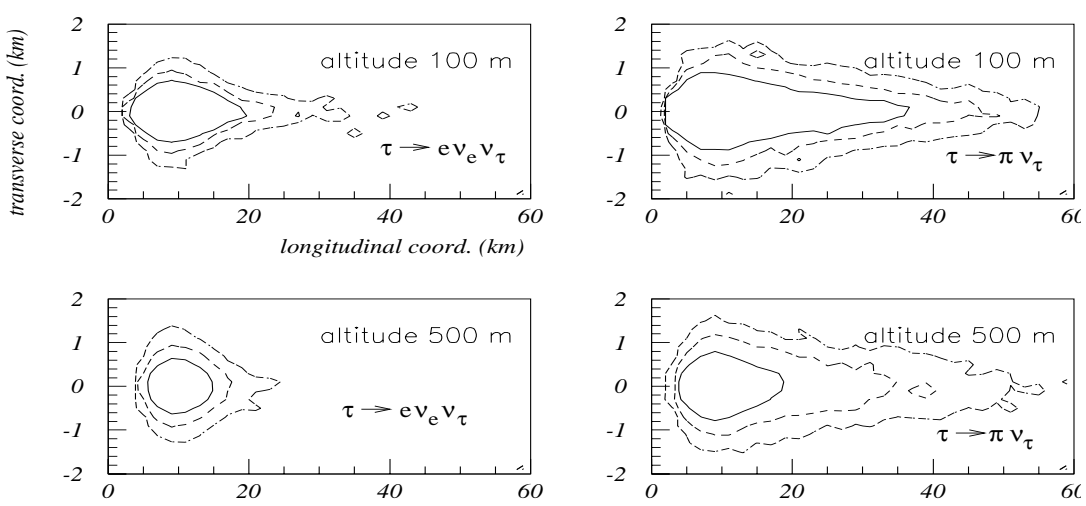

Figure 2: Ground spots of horizontal showers induced by a $\tau$ of $1 \mathrm{EeV}$. Lines indicate the level $L$ of the tank local trigger (solid), $L / 3$ (dashed) and $L / 10$ (dotted). The event is seen if the inner area is typically $8 \mathrm{~km}^{2}$ or more. With large muonic component, hadronic decay is favoured.

\section{Tau Event Simulation}

A $\tau$ lepton may interact several times through deep inelastic scattering, changing charge in most cases, and eventually decay. Some energy is lost at each interaction, as well as continuously along the path. At UHE, the initial direction is always conserved (Figure (3i).

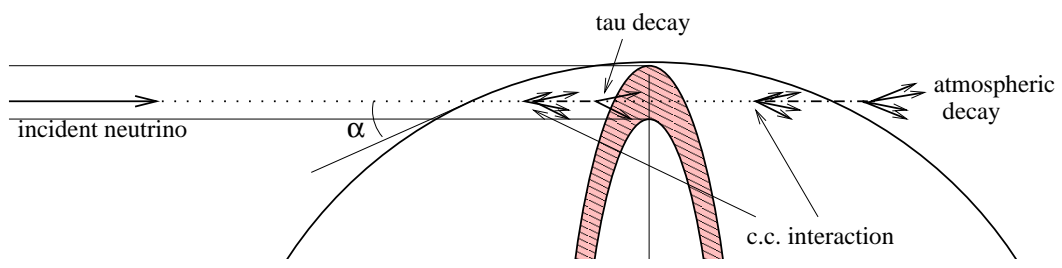

Figure 3: Chain of interactions producing an observable shower.

We assume an isotropic incident flux, an homogeneous Earth with density $2.15 \mathrm{~g} / \mathrm{cm}^{3}$, and a charged current (CC) cross section as given by the results of the CTEQ4-DIS parton distributions : $\sigma_{c c}^{\nu N}=1.0\left(E_{\nu} / 1 \mathrm{EeV}\right)^{0.363} 10^{-32} \mathrm{~cm}^{2}$. Neutral current interactions are also taken into account, with $\sigma_{n c}=0.4 \sigma_{c c}[\overline{8}]$.

Energy losses of the form $d E / d x=a+b(E) E(\simeq b E$ at UHE) are calculated including Bremsstrahlung (BS) and Pair Production (PP) as well as Deep Inelastic Scattering on nuclei (DIS). Contributions from BS and PP have been rescaled from the muon values

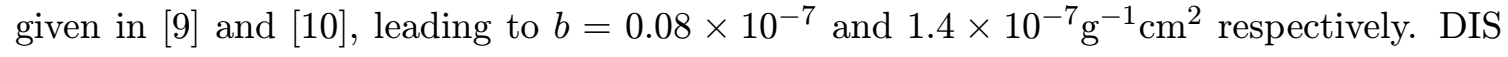
contributions rely on parameterization of the photo-nucleon cross sections as well as on the proper modelisation of the nucleon structure functions at very low $x$ and/or very large $Q^{2}$. Here we try two estimates of $b$ : an constant value (DIS-low, $b=10^{-7} \mathrm{~g}^{-1} \mathrm{~cm}^{2}$ ) rescaled from the muon behavior given in [i] $\left.b=6 E_{18}^{0.2} 10^{-7} \mathrm{~g}^{-1} \mathrm{~cm}^{2}\right)$ as a parameterisation of the recent calculation from [i]

The atmospheric shower (if any) is generated with AIRES [i] is then evaluated through a detailed simulation of the interactions of incident particles in 
water. A standard selection is applied: at least 4 stations hit within $20 \mu$ s at the level of $4 \mathrm{vem}$ (vertical equivalent muons), in a relatively compact topology.

The probability to detect a shower with a given visible energy depends mainly on the altitude of the core $10 \mathrm{~km}$ after the decay, not on the precise trigger conditions (see Fig., $\frac{\overline{4}}{4}$ ).

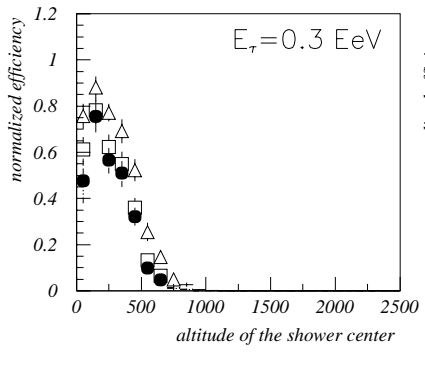

trigger condition : $\triangle 3$ tanks

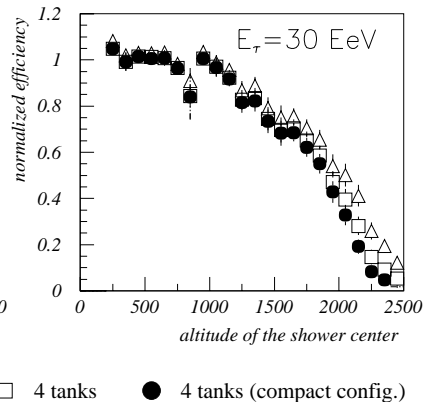

$\square \quad 4$ tanks

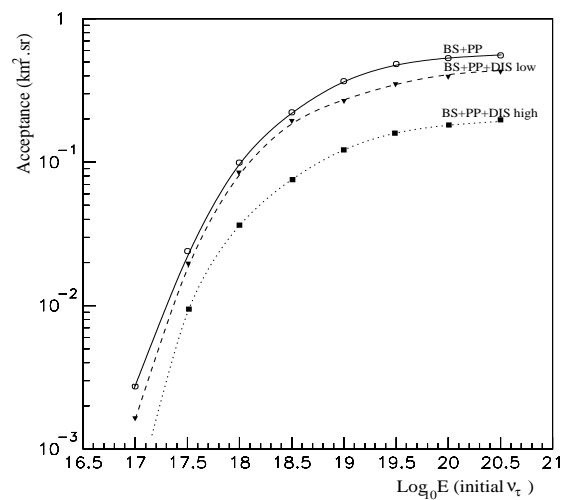

Figure 4: Left: probability of $\tau$ detection vs altitude (with different trigger conditions). Right: acceptance, with various energy loss models; BS : bremmstrahlung, PP pair production, DIS deep inelastic scattering.

Neutrino terrestrial interactions may be distinguished from atmospheric ones because they are concentrated at $\sin \theta \simeq 1$. However, the energy of the primary neutrino cannot be accurately known, because of unpredictable losses in intermediate steps and through secondary neutrinos from $\tau$ decay; moreover the altitude of the decay is a priori unknown.

\section{Expected event rate}

The Auger sensitivity is plotted in Fig. given in Table $i_{-i}^{1}$. For atmospheric interactions, only the models classified

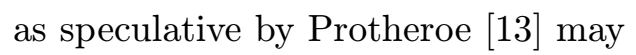
yield a detectable signal, while $\tau$ from earth may be seen even for the lowest expected fluxes.

\begin{tabular}{l|c|c|c|c|c} 
DIS & AGN-1 & TD & GRB & GZK & AGN-2 \\
\hline none & 27.0 & 2.3 & 0.5 & 1.7 & 2.9 \\
\hline low & 24.0 & 1.8 & 0.4 & 1.5 & 2.5 \\
\hline high & 10.0 & 0.8 & 0.2 & 0.6 & 1.1
\end{tabular}

Table 1: Annual rates for the source models presented in Fig. energy losses.

\section{Conclusion}

The Surface Detector of the Auger Observatory is found to be able to detect $\nu_{\tau}$ interactions in earth in the $0.1-10 \mathrm{EeV}$ range, through horizontal showers close to the ground. A fluorescence signal may be seen at higher altitude, but not far away, and the duty time or the telescope is about $10 \%$ : hybrid detection is expected to be very rare.

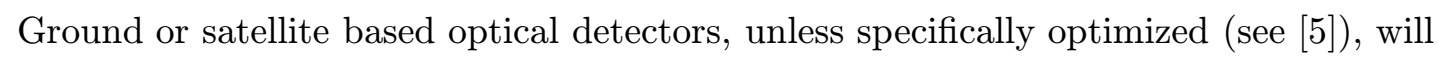
not be able to observe efficiently those events. This makes Auger the only available observatory able to probe the GZK energy window. 


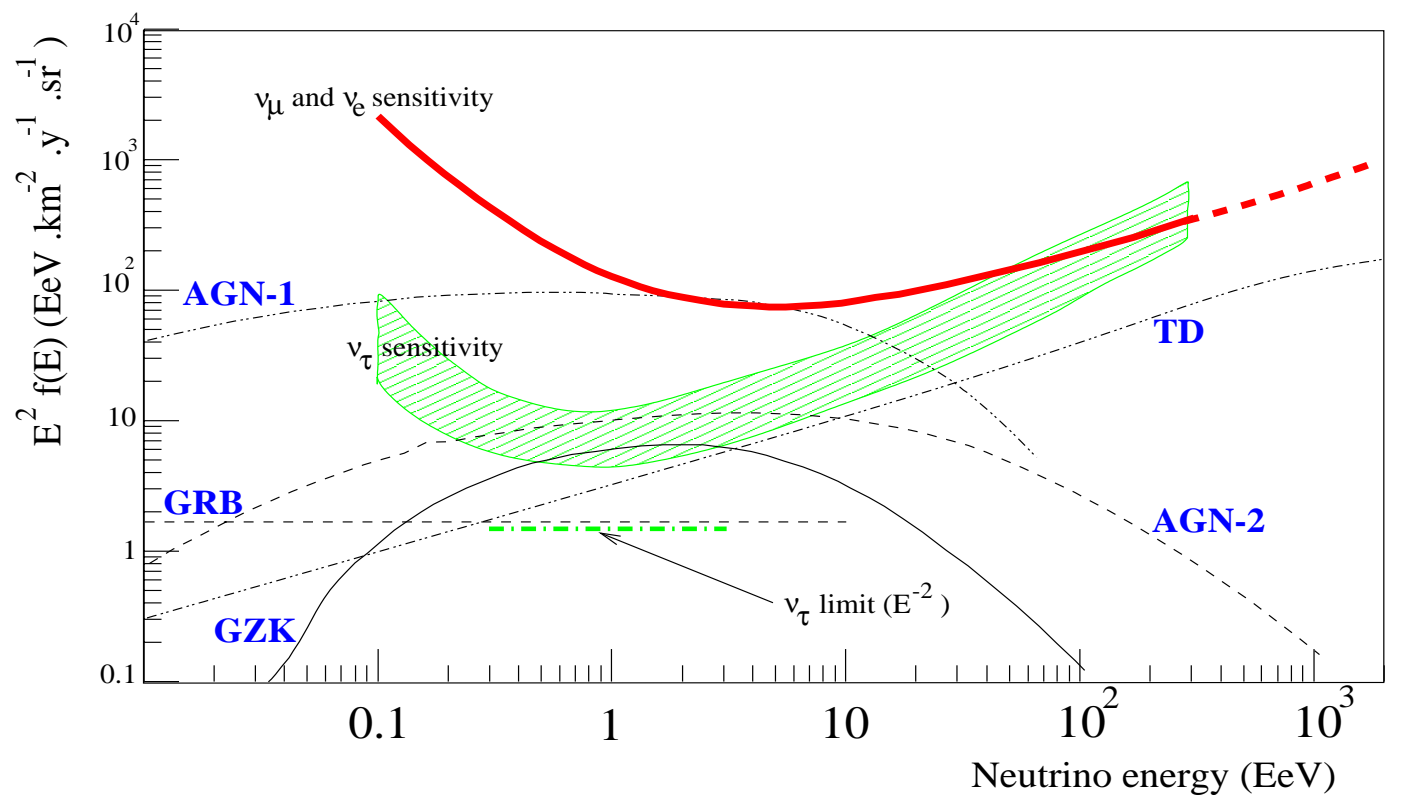

Figure 5: Muon or tau neutrino fluxes from various sources in the full mixing hypothesis, taken from [13]. Dotted lines are speculative fluxes, dashed probable and solid almost certain. The thick solid line and the hatched area represent the Auger sensitivity (flux producing one event per year and per decade). Top line for horizontal shower from $\nu_{e}$ and $\nu_{\mu}$ interactions in the atmosphere [4] hatched area for $\nu_{\tau}$, depending on DIS loss. We also plotted the $90 \%$ C.L. limit after five years for an $\mathrm{E}^{-2}$ flux between 0.3 and $3 \mathrm{EeV}$.

\section{References}

[1] K. Greisen, Phys. Rev. Lett. 16 (1966)748.; G.T.Zatsepin, V.A.Kuzmin, JETP Lett. 4 (1966) 78.

[2] The Pierre Auger Project Design Report, Fermilab (1995), www.auger.org/admin/.

[3] G. Parente, E. Zas, astro-ph/ 9606091; K. S. Capelle, J. W. Cronin, G. Parente, E. Zas, Astropart. Phys. 8 (1998) 321; S. Coutu, X. Bertou, P. Billoir, John Hopkins Workshop (Neutrinos in the Next Millenium), 1999 (sub. to World Scienc.).

[4] P. Billoir, 8th International Workshop on Neutrino Telescopes, (1999) 111.

[5] D. Fargion, astro-ph/ 0002453 and astro-ph/ 0101565.

[6] A. Letessier-Selvon, astro-ph/ 0009444.

[7] S. Fukuda et al., Phys. Rev. Lett. 81, (1998) 156; and updates in C. McGrew, "9th International Workshop on Neutrino Telescopes", Venice (Italy), March 6-9 (2001).

[8] R. Gandhi, C. Quigg, M. H. Reno and I. Sarcevic, Phys. Rev. D 58 (1998).

[9] Particle Data Group, D.E. Groom et al., European Physical Journal C15, (2000) 1.

[10] A.V Ginneken Nucl. Instrum. Methods A251, (1986) 21.

[11] S.I. Dutta, M.H. Reno, I. Sarcevic and D. Seckel, Phys.Rev. D63, (2001) 094020; also in hep-ph/0012350.

[12] S.J. Sciutto, AIRES, a system for air shower simulations, version 2.2.1 (2000).

[13] R.J. Protheroe, astro-ph/ 9809144. 